\title{
Defect luminescence in undoped p-type GaSe
}

\section{A. Aydinli}

To cite this article: A. Aydinli (2001) Defect luminescence in undoped p-type GaSe, Philosophical Magazine Letters, 81:12, 859-867, DOI: 10.1080/09500830110093885

To link to this article: https://doi.org/10.1080/09500830110093885

Published online: 14 Nov 2010.

Submit your article to this journal $2 \pi$

III Article views: 43

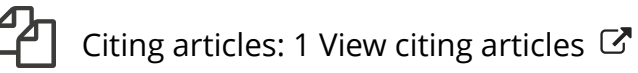




\title{
Defect luminescence in undoped p-type GaSe
}

\author{
A. Aydinli $\dagger$ \\ Department of Physics, Bilkent University, Ankara 06533, Turkey
}

N. M. Gasanly $\ddagger$ and K. GöKŞEN

Department of Physics, Middle East Technical University, Ankara 06531, Turkey

[Received in final form 17 August 2001 and accepted 22 August 2001]

\begin{abstract}
Photoluminescence (PL) spectra of undoped single crystals of the layered semiconductor GaSe have been measured in the temperature range from $10 \mathrm{~K}$ to room temperature and in the wavelength range from 635 to $750 \mathrm{~nm}$. Two wide bands centred at 644 and $695 \mathrm{~nm}$ have been observed at $T=10 \mathrm{~K}$. A detailed analysis of the spectra obtained by varying the excitation intensity and temperature resulted in the identification of the levels involved. A simple model is proposed to account for the observed data.
\end{abstract}

\section{$\S 1$. INTRODUCTION}

GaSe is a group III-VI semiconductor that crystallizes in a layered structure which contains four monatomic sheets in the order $\mathrm{Se}-\mathrm{Ga}-\mathrm{Ga}-\mathrm{Se}$. A single layer is hexagonally ordered and the $\mathbf{c}$ axis is perpendicular to the layers. Depending on the period of stacking sequences there are four modifications: $\varepsilon, \beta, \gamma$ and $\delta$ (Kuhn et al. 1975). The $\varepsilon$ and $\beta$ modifications are $2 \mathrm{H}$ hexagonal type, whereas $\gamma$ and $\delta$ are $3 \mathrm{R}$ trigonal type. Most growth techniques result in crystals of $\varepsilon$ and $\gamma$ type. In all these modifications, the intralayer chemical bondings are predominantly covalent while the interlayer bonding is relatively weak and van der Waals like.

The electronic band structure of GaSe shows an indirect minimum of the conduction band at the $\mathrm{M}$ point of the Brillouin zone which is lower than the direct gap at the $\Gamma$ point. Optical transitions between the valence band and the direct and the indirect minima in the conduction band are allowed with various strengths depending on the polarization of the light. The indirect gap energy in GaSe is $2.102 \mathrm{eV}$ at 4.2 K (Mercier and Voitchovsky 1975).

Considerable work has been carried out on the study of photoluminescence (PL) from GaSe. The low-temperature PL spectrum of GaSe single crystals is quite complex and shows a large number of sharp lines near the band edge and wider bands away from the band edge. Band-edge luminescence in GaSe is very rich in the number of lines observed. This is because, in GaSe, the indirect minimum of the conduction band is $25 \mathrm{meV}$ lower than the direct minimum. As a result of this proximity, photoexcited carriers populate both bands and give rise to many lines owing to radiative recombinations related to these bands. Both direct and indirect

$\dagger$ Author for correspondence. Email: aydinli@fen.bilkent.edu.tr

$\ddagger$ On leave from Department of Physics, Baku State University, Baku, Azerbaijan.

Philosophical Magazine Letters ISSN 0950-0839 print/ISSN 1362-3036 online (C) 2001 Taylor \& Francis Ltd 
free and bound excitonic structure, as well as their phonon replicas, are observed (Capozzi 1981).

Wide bands observed at lower energies are attributed to intrinsic defects in the case of undoped samples and to impurities and defects for doped samples. For undoped $\varepsilon$-GaSe (Capozzi and Montagna 1989), increasing the concentration of defects has been shown to lead to increasing PL intensity as well as a blue shift of the band. PL spectra of undoped samples have been used to compare with the PL spectra of Tm-doped GaSe and three bands at 590, 610 and $690 \mathrm{~nm}$ have been observed in undoped GaSe (Tagiev et al. 1985). Both excitonic and impurity-related emission lines have been observed in GaSe (Voitchovsky and Mercier 1974) at $4.2 \mathrm{~K}$ in the range $580-610 \mathrm{~nm}$ and, since no recombination radiation specific to the introduced dopant had been detected, donor and acceptor states taking place in the recombination were thought to have originated from structural defects whose concentration increased with increasing impurity content. On the other hand, PL from both undoped and $\mathrm{Cu}$-doped $\mathrm{GaSe}$ at $80 \mathrm{~K}$ have been studied (Capozzi 1983) and donor states related to structural defects as well as acceptor states related to $\mathrm{Cu}$ have been proposed. Several PL studies involving various impurities such as Cd (Shigetomi et al. 1991), Ag (Shigetomi et al. 1997), Mn (Tagiev et al. 1984), Zn (Shigetomi et al. 1993), Cu, Zn, Cd, Sn, and I (Schmid et al. 1974) have also revealed both excitonic and impurity-related lines in GaSe.

In spite of all these data, PL spectra of undoped GaSe away from the excitonic region are not well characterized at low temperatures. In particular, no systematic analysis of the defect-related PL spectra of undoped GaSe exists in the literature to our knowledge. In this work, we present PL data on Bridgman-grown undoped GaSe in the wavelength region $635-750 \mathrm{~nm}$ at temperatures as low as $10 \mathrm{~K}$. We systematically studied the temperature $(10-300 \mathrm{~K})$ and excitation intensity $\left(4.8 \times 10^{-3}-4.30 \mathrm{~W} \mathrm{~cm}^{-2}\right)$ dependences of the PL spectra from undoped GaSe and propose an energy band diagram to explain the results.

\section{$\S 2$. Experimental details}

GaSe polycrystals were synthesized from high-purity elements (at least $99.999 \%$ pure) prepared in stoichiometric proportions. Single crystals of $\varepsilon$-GaSe were grown by the modified Bridgman method. The analysis of X-ray diffraction data showed that GaSe crystallizes in a hexagonal unit cell with lattice parameters $a=0.3745 \mathrm{~nm}$ and $c=1.5921 \mathrm{~nm}$. The samples were prepared by cleaving an ingot parallel to the crystal layer, which was perpendicular to the c axis with typical sample dimensions of $4 \mathrm{~mm} \times 3 \mathrm{~mm} \times 0.7 \mathrm{~mm}$. The electrical conductivity of the studied samples was $p$ type as determined by the hot-probe method. A Spectra-Physic s He-Ne laser and an Ar-ion laser operating at wavelengths of $632.8,514.5$ and $488.0 \mathrm{~nm}$ were used as the excitation sources. The PL was observed from the laser-illuminated face of the samples, in a direction close to the normal of the (001) plane. A CTI-Cryogenics M-22 closed-cycle He cryostat was used to cool the crystals from room temperature to $10 \mathrm{~K}$. The surface of the sample was freshly cleaved just prior to loading it on to the cold finger of the closed-cycle refrigerator. The temperature was controlled within an accuracy of $\pm 0.5 \mathrm{~K}$. The PL spectra in the $635-750 \mathrm{~nm}$ wavelength range were analysed using a Jobin-Yvon U-1000 double-grating spectrometer and a cooled GaAs photomultiplier equipped with the necessary photon-counting electronics. All spectra have been corrected for the spectral response of the optical 
apparatus. A set of neutral-density filters was used to adjust the excitation laser intensity from $4.8 \times 10^{-3}$ to $4.30 \mathrm{~W} \mathrm{~cm}^{-2}$.

\section{$\S 3$. RESUlTS AND Discussion}

We first used the 488.0 and $514.5 \mathrm{~nm}$ wavelengths of the Ar-ion laser to excite PL in GaSe. For both wavelengths, this results in intense near-band-edge emission due to recombination of direct and indirect free and bound excitons which are well characterized in GaSe. No other features have been observed beyond $635 \mathrm{~nm}$. These results are in agreement with those previously obtained (Shigetomi et al. 1991). In fact, it has long been recognized that the observation of non-excitonic emission in GaSe requires a low pumping power, high impurity content or very low temperatures (Voitchovsky and Mercier 1974). Alternatively, longer excitation wavelengths can also be used to try to observe non-excitonic PL from GaSe (Gnatenko et al. 1990). In the present work, we concentrate on non-excitonic PL spectra obtained by excitation with $632.8 \mathrm{~nm}$ line of a He-Ne laser.

Figure 1 shows the typical non-excitonic PL spectra obtained from GaSe single crystals as measured in the $635-750 \mathrm{~nm}$ wavelength range and $10-150 \mathrm{~K}$ temperature range at a constant excitation intensity of $4.30 \mathrm{~W} \mathrm{~cm}^{-2}$. We observe two broad bands

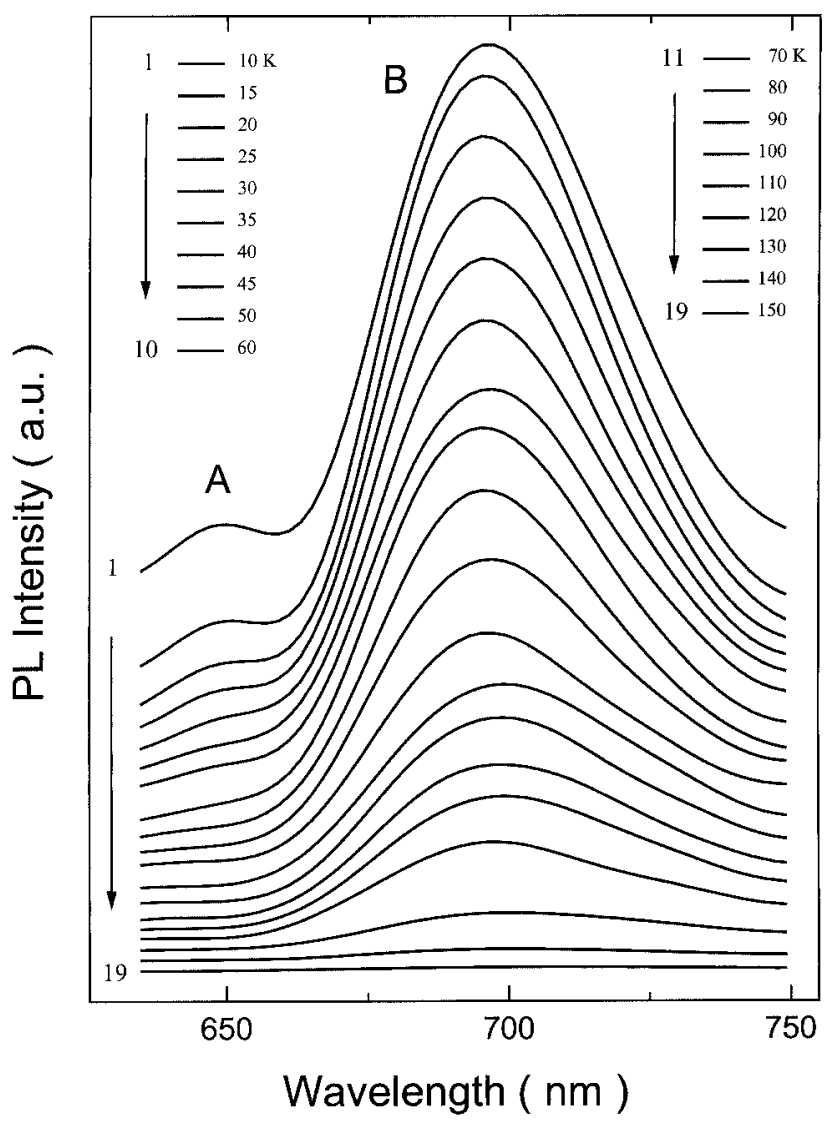

Figure 1. PL spectra of undoped p-type GaSe as a function of temperature at $L=4.30 \mathrm{~W} \mathrm{~cm}^{-2}$ (a.u., arbitrary units). 
labelled as A and B bands. The A band is lower in intensity while the intensity of the $\mathrm{B}$ band dominates the spectrum. We deconvoluted the spectra with two Gaussian peaks using a least-squares approach and found that the PL bands are centred at $644 \mathrm{~nm}(1.925 \mathrm{eV})$ and $695 \mathrm{~nm}(1.784 \mathrm{eV})$ at $10 \mathrm{~K}$. We note that both the PL intensity and the PL peak energy change as functions of increasing sample temperature. As the temperature is increased, the PL intensities of both bands decrease. All PL activity ceases to be observed at $150 \mathrm{~K}$. The observed bands have Gaussian line shapes and half-widths of 0.078 and $0.110 \mathrm{eV}$ for the A and B bands, respectively. These features are typical of donor-acceptor pair recombination processes in semiconductors (Leyris et al. 1983).

From figure 1, it can be seen that the intensities of both bands decrease with increasing temperature with the A band quenching much more rapidly than the $\mathrm{B}$ band. The variations in the PL peak intensities of both bands with respect to temperature are plotted in figure 2. In the low-temperature range, the PL intensities of both bands decrease slowly. Above $35 \mathrm{~K}$ for the $\mathrm{A}$ band and $90 \mathrm{~K}$ for the $\mathrm{B}$ band, the rates of intensity decrease for both bands increase significantly owing to thermal quenching processes. The activation energies for both bands were obtained by fitting the high-temperature regions of the data to the following equation:

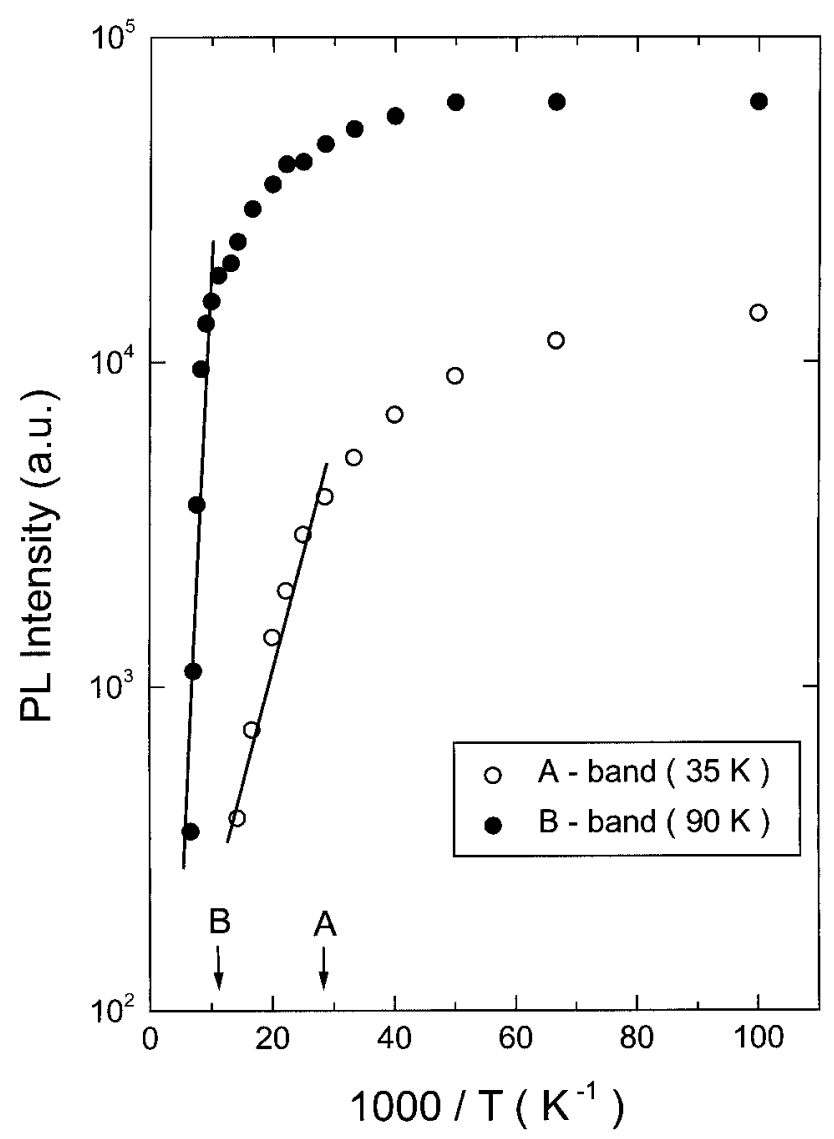

Figure 2. Temperature dependences of GaSe PL intensity at the emission band maxima (a.u., arbitrary units). The arrows at 35 and $90 \mathrm{~K}$ show the starting points of the intensive quenching of the $\mathrm{A}$ and $\mathrm{B}$ bands respectively. 


$$
I=I_{0} \exp \left(\frac{\Delta E}{k_{\mathrm{B}} T}\right),
$$

where $I$ is the PL intensity, $I_{0}$ is a proportionality constant and $k_{\mathrm{B}}$ is Boltzmann's constant. The semilogarithmic plots of the peak intensity as a function of the reciprocal temperature give straight lines in the $35-70$ and $90-150 \mathrm{~K}$ regions for the A and $\mathrm{B}$ bands respectively. The slopes of these lines give the activation energies as $0.014 \mathrm{eV}$ for the A band and $0.076 \mathrm{eV}$ for the B band. Since the as-grown GaSe is a ptype semiconductor, as confirmed by the measurement of the carrier type using the hot-probe method, we assume that the activation energies that we obtained from figure 2 correspond to the activation energies of the acceptor levels. Similar assignments have been made for Ag-doped (Shigetomi et al. 1997) and Cu-doped (Capozzi 1983 ) p-type GaSe. The activation energy of $0.076 \mathrm{eV}$ obtained for the B band can be compared with previous data obtained using various measurement techniques. From the temperature dependence of the dark conductivity in undoped p-type GaSe, acceptor levels of $0.075 \mathrm{eV}$ (Tatsuyama et al. 1971), $0.086 \mathrm{eV}$ (Manfredotti et al. 1975 ) and $0.070 \mathrm{eV}$ (Ismailov et al. 1966) above the valence band were deduced. On the other hand, the temperature dependence of the Hall coefficient has yielded a value of $0.066 \mathrm{eV}$ (Manfredotti et al. 1977). The value of $0.076 \mathrm{eV}$ obtained by us using the temperature dependence of the PL intensity is in fair agreement with these data. The activation energy of $0.014 \mathrm{eV}$ that we obtained for the $\mathrm{A}$ band corresponds to the ionization energy of a shallow level above the top of the valence band maximum and could not have been previously obtained since, to our knowledge, all measurements on GaSe were made and analysed at $80 \mathrm{~K}$ or higher.

We studied the temperature dependence of the peak energy of each band. PL emissions obtained from the A and B bands quench totally above 70 and $150 \mathrm{~K}$ respectively. The A band shifts towards the red very slightly beyond $50 \mathrm{~K}$. The $\mathrm{B}$ band also shows a small red shift, starting at $50 \mathrm{~K}$ and becoming larger beyond $100 \mathrm{~K}$. Since for GaSe the rate of change in the bandgap energy with increasing temperature is negative, the observed red shifts are to be expected.

The behaviours of both bands as a function of excitation laser intensity were also studied. We found that the peak energy of the A band does not shift with increasing excitation laser intensity in contrast with the B band. This may be due to a homogeneous distribution of donor-acceptor pairs involved in the emission of the A band. The dependence of the emission peak energy $E_{\mathrm{p}}$ of the $\mathrm{B}$ band at $10 \mathrm{~K}$ as a function of excitation laser energy intensity $L$ is given in figure 3 . Inspection of the data shows that the maximum of this band shifts towards higher energies from 1.770 to $1.784 \mathrm{eV}$ $\left(\Delta E_{\mathrm{p}}=0.014 \mathrm{eV}\right)$ with increasing excitation laser intensity from $4.8 \times 10^{-3}$ to $4.30 \mathrm{~W} \mathrm{~cm}^{-2}$ (i.e. $4.8 \mathrm{meV}$ per decade of intensity of exciting radiation). This shift is relatively small because of the limited range of excitation laser intensity available from our He-Ne laser. The observed blue shift is characteristic of the donoracceptor pair recombination process. The magnitude of the observed blue shift is of the same order as in other binary and ternary semiconductor compounds such as ZnSe, GaP (Zacks and Halperin 1972), InP (Montie and van Gurp 1989), GaAs (Pankove 1971), TlGaSe 2 (Gasanly et al. 2000) and $\mathrm{CuGaSe}_{2}$ (Poure et al. 1981), which are 3.2, 3.6, 2-10, 8.8, 6.0 and $6.8 \mathrm{meV}$ per decade of exciting radiation intensity respectively. In donor-acceptor pair recombination, photoexcited carriers trapped at donors and acceptors recombine radiatively. The energy of the emitted radiation due to recombination of carrier trapped at donor and acceptor sites is given as 


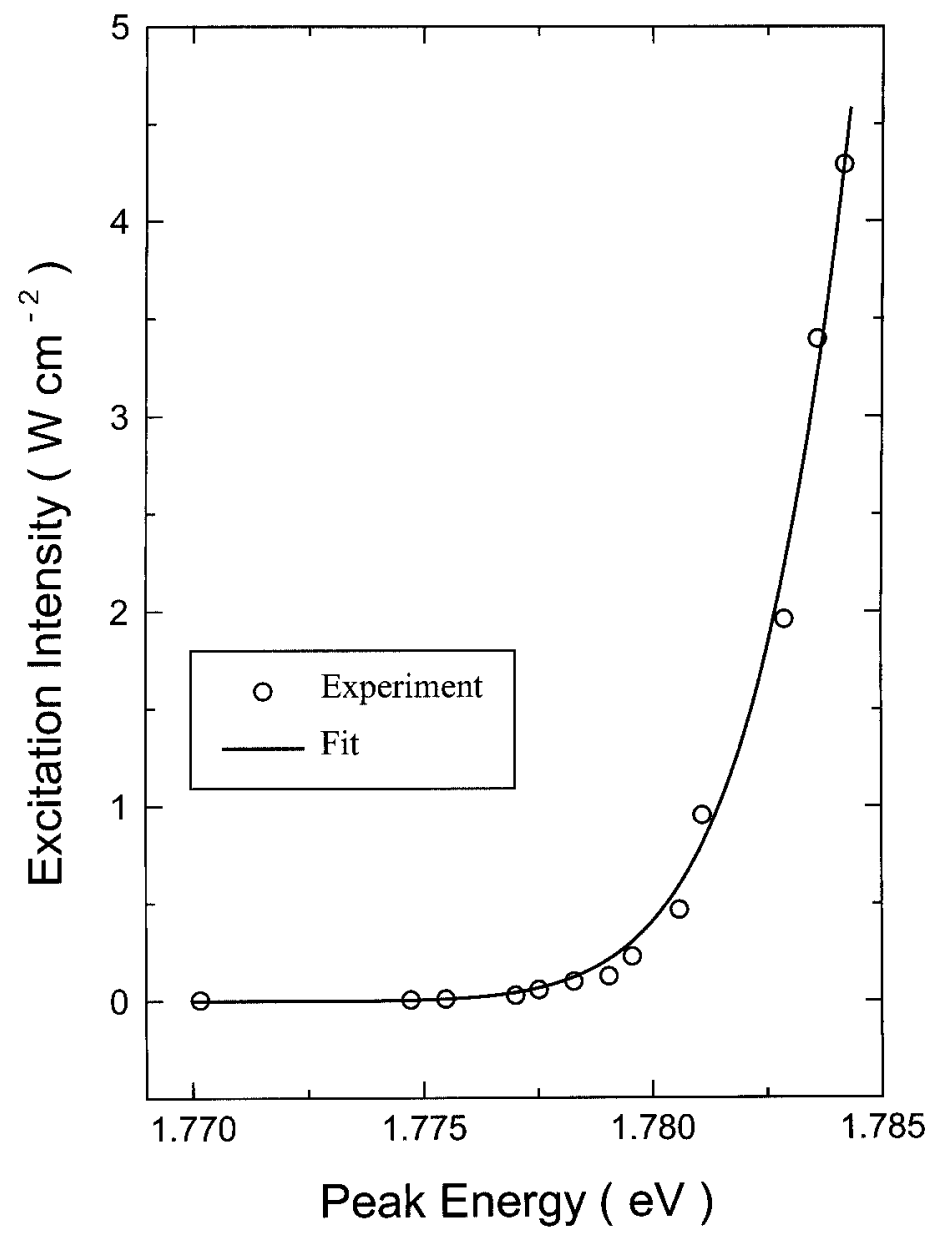

Figure 3. Excitation laser intensity versus GaSe emission B-band peak energy at $10 \mathrm{~K}$ : (-), theoretical fit using equation (3).

$$
E(\hbar \omega)=E_{\mathrm{g}}-E_{\mathrm{D}}-E_{\mathrm{A}}-\frac{Z_{\mathrm{D}} Z_{\mathrm{A}} e^{2}}{\varepsilon R},
$$

where $E_{\mathrm{g}}$ is the bandgap energy of GaSe, $E_{\mathrm{D}}$ is the donor level energy, $E_{\mathrm{A}}$ is the acceptor level energy, $R$ is the distance between pairs, $Z_{\mathrm{D}}$ and $Z_{\mathrm{A}}$ are the charge states of the donor and acceptor sites respectively and $\varepsilon$ is the dielectric constant. As can be seen from the above equation, only distant pairs are excited at low excitation laser intensities (Pankove 1971). At higher excitation intensities, closer pairs are also excited and contribute to the observed radiative intensity. On the other hand, Coulombic interaction between ionized sites is a function of the distance between the pairs and makes a positive contribution to the energy of the emitted photon. This contribution increases as the separation between the pairs decreases $(\mathrm{Yu}$ and Cardona 1996). Furthermore, the radiative transition probabilities for different pair separations are not the same and decrease exponentially as a function of the pair distance (Leyris et al. 1983). We used the following expression to fit the experimental data in figure 3 : 


$$
L\left(E_{\mathrm{p}}\right)=L_{0} \frac{\left(E_{\mathrm{p}}-E_{\infty}\right)^{3}}{E_{\mathrm{B}}+E_{\infty}-2 E_{\mathrm{p}}} \exp \left(-\frac{2\left(E_{\mathrm{B}}-E_{\infty}\right)}{E_{\mathrm{p}}-E_{\infty}}\right)
$$

where $L_{0}$ is a proportionality constant, $E_{\mathrm{B}}$ is the emitted photon energy of a close donor-acceptor pair separated by a shallow-impurity Bohr radius $R_{\mathrm{B}}$ and $E_{\infty}$ is the emitted photon energy of an infinitely distant donor-acceptor pair (Zacks and Halperin 1972). From a nonlinear least-squares fit to the experimental data, the photon energies of an infinitely distant donor-acceptor pair and a close donoracceptor pair separated by $R_{\mathrm{B}}$ are found to be $E_{\infty}=1.762 \mathrm{eV}$ and $E_{\mathrm{B}}=1.832 \mathrm{eV}$ respectively. These limiting photon energies are in good agreement with the bandgap energy $\left(E_{\mathrm{g}}=2.102 \mathrm{eV}\right)$ and the observed values of the peak energy position (i.e. $E_{\infty}<1.770 \mathrm{eV}<E_{\mathrm{p}}<1.784 \mathrm{eV}<E_{\mathrm{B}}<E_{\mathrm{g}}$ ) at $10 \mathrm{~K}$.

We also investigated the intensity variation of the maximum of the emission band versus the excitation laser intensity at $T=10 \mathrm{~K}$ (figure 4). The experimental data can be fitted by a simple power law

$$
I \propto L^{\gamma}
$$

where $I$ is the PL intensity, $L$ is the excitation laser intensity and $\gamma$ is a dimensionless exponent. It was found that the PL intensity increases sublinearly and yields values

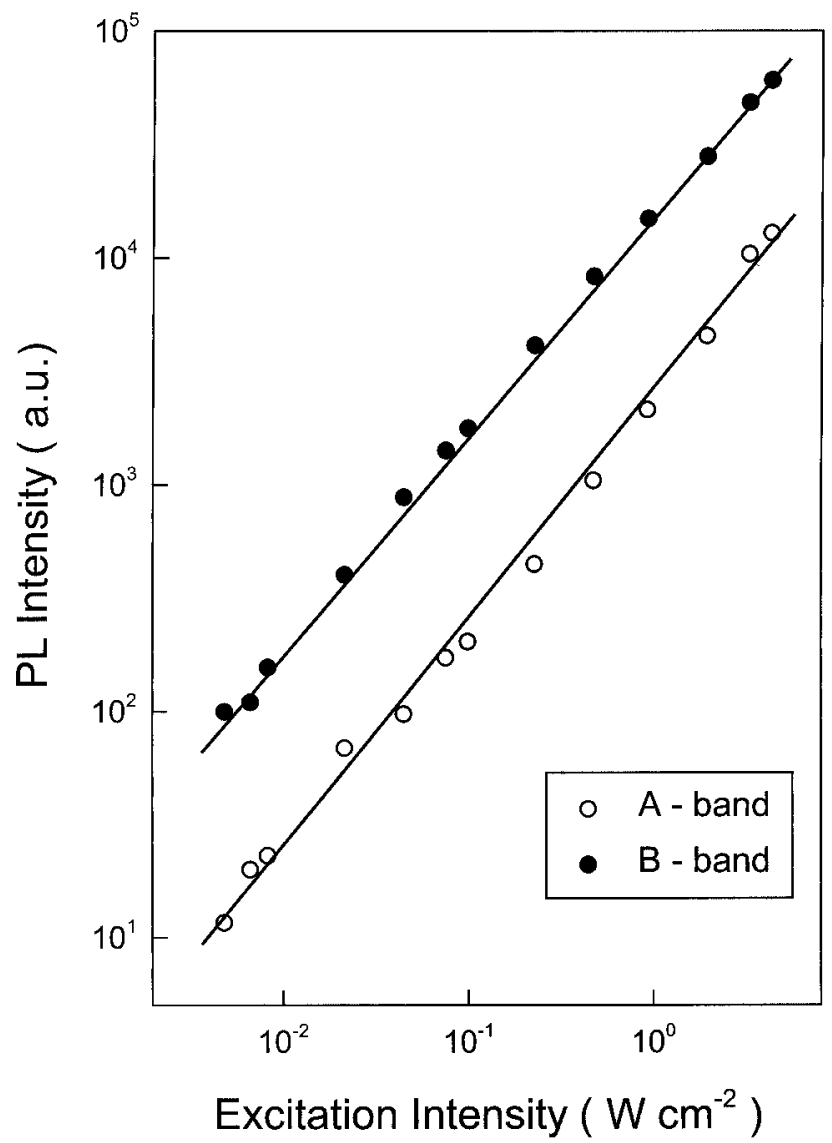

Figure 4. Dependence of GaSe PL intensity at the emission band maxima versus excitation laser intensity at $10 \mathrm{~K}$ (a.u., arbitrary units): (-), theoretical fits using equation (4). 
of $\gamma=0.97$ and $\gamma=0.95$ for the A and B bands respectively. No saturation of the PL intensity was observed in the highest excitation laser intensity available to us. For an excitation laser energy larger than the bandgap energy $E_{\mathrm{g}}$ of a semiconductor, the coefficient $\gamma$ is generally $1<\gamma<2$ for free and bound exciton emission, and $\gamma \leqslant 1$ for free-to-bound and donor-acceptor pair recombination (Schmidt et al. 1992). Thus the obtained value of $\gamma<1$ for both bands is further evidence that the observed emission is due to donor-acceptor pair recombination.

The analysis of the observed PL data as a function of temperature and excitation laser intensity allows us to propose a possible model for the donor-acceptor levels located in the forbidden gap of GaSe. These levels are involved in the radiative recombination of photoexcited carriers. In the model that we propose, the activation energies obtained from the temperature-dependent intensities of the A and B bands suggest the presence of two acceptor levels $\mathrm{a}_{1}$ and $\mathrm{a}_{2}$ which are located 0.014 and $0.076 \mathrm{eV}$ respectively above the valence band. On the other hand, using the general expression for the emission energy of the donor-acceptor pair and taking into account $E_{\mathrm{g}}$ and $E_{\infty}$, the sum of the activation energy $E_{\mathrm{d}}$ of the donor level and the activation energy $E_{\mathrm{a}}$ of the acceptor level involved in the emission band can be estimated as

$$
E_{\mathrm{a}}+E_{\mathrm{d}}=E_{\mathrm{g}}-E_{\infty}=2.102-1.762 \mathrm{eV}=0.340 \mathrm{eV} .
$$

Considering that the acceptor level is located at $0.076 \mathrm{eV}$ above the top of the valence band, this result suggests that the donor level $d_{2}$ involved in the emission of the $B$ band is located $0.264 \mathrm{eV}$ below the bottom of the conduction band. For the A band, the shallow acceptor level at $0.014 \mathrm{eV}$ and the observed peak energy at $10 \mathrm{~K}$ $(1.925 \mathrm{eV})$, results in a donor level $\mathrm{d}_{1}$, which is located $0.163 \mathrm{eV}$ below the bottom of the conduction band. We propose that these moderately deep donor and shallow acceptor levels are associated with stacking faults or dislocations, which are easily produced in these materials owing to weak van der Waals forces between the layers.

Moderately deep donor levels were suggested in nominally undoped p-type GaSe layered crystals previously (Capozzi 1983). There, in addition to excitonic features, four weak bands centred at $645 \mathrm{~nm}(1.923 \mathrm{eV}), 662 \mathrm{~nm}(1.872 \mathrm{eV}), 700 \mathrm{~nm}(1.771 \mathrm{eV})$ and $742 \mathrm{~nm}(1.672 \mathrm{eV})$ were observed at $80 \mathrm{~K}$. Considering that $\mathrm{d} E_{\mathrm{g}} / \mathrm{d} T=-0.47 \mathrm{meV} \mathrm{K}^{-1}$ for GaSe, a blue shift of $33 \mathrm{meV}$ should be expected for these lines at $10 \mathrm{~K}$. Two of these lines $(1.923$ and $1.672 \mathrm{eV})$ were assigned to boundto-free transitions from two donor levels to the valence band. The donor levels were located 0.170 and $0.420 \mathrm{eV}$ below the bottom of the conduction band. The donor level located at $0.170 \mathrm{eV}$ below the conduction band agrees closely with our finding of a donor level at $0.163 \mathrm{eV}$ below the conduction band. The other two transitions were thought to be due to donor-acceptor pair transitions from the first donor level to acceptor levels at 0.051 and $0.152 \mathrm{eV}$ above the valence-band maximum. In a later study (Capozzi and Montagna 1989), only two bands at 1.88 and $1.62 \mathrm{eV}$ were observed at $80 \mathrm{~K}$ from nominally undoped p-type GaSe beyond $635 \mathrm{~nm}$. No activation energies of impurity levels involved in the emission were obtained from the temperature dependence of emitted intensities. In contrast with their earlier work, they assigned the high-energy band to a transition from a shallow donor $\left(E_{\mathrm{D}}=0.006 \mathrm{eV}\right)$ to an acceptor level $\left(E_{\mathrm{A}}=0.20 \mathrm{eV}\right)$ and the low-energy band to recombination of free electrons with deep neutral acceptors $\left(E_{\mathrm{A}}=0.44 \mathrm{eV}\right)$, using values published in the literature for donor and acceptor level activation energies obtained from electrical measurements. While the number of bands and the approxi- 
mate spectral location of these bands that we observe are the same as those observed in their work, our conclusions and, except for the donor level at $0.163 \mathrm{eV}$, the initial and final states that are involved in these transitions are different. This may be due to the possibility of several donor and acceptor states, both deep and shallow, in undoped GaSe. The presence of any number of these states in a given GaSe crystal may depend on the details of the growth conditions of each crystal.

\section{$\S 4$. Conclusions}

Non-excitonic PL spectra of undoped GaSe were studied as a function of temperature and excitation laser intensity. Two broad bands at 644 and $695 \mathrm{~nm}$ were observed at $10 \mathrm{~K}$. Considering the p-type behaviour of undoped $\mathrm{GaSe}$, and the activation energies obtained from the temperature dependence of the emitted intensities, we deduce two shallow acceptor levels at 0.014 and $0.076 \mathrm{eV}$ above the top of the valence-band maximum. From the peak energy positions of the bands, we infer the existence of two moderately deep donor levels at 0.163 and $0.264 \mathrm{eV}$ below the bottom of the conduction band. We therefore propose that the bands that we observe are due to carrier recombination through neutral donor-acceptor pairs.

\section{REFERENCES}

Capozzi, V., 1981, Phys. Rev. B, 23, 836; 1983, Ibid., 28, 4620.

Capozzi, V., and Montagna, M., 1989, Phys. Rev. B, 40, 3182.

Gasanly, N. M., Serpenguzel, A., Aydinli, A., and Baten, S. M. A., 2000, J. Lumin., 86, 39.

Gnatenko, Y. P., Zhirko, Y. I., and Kovalyuk, Z. D., 1990, Phys. Stat. sol. (b), 161, 427. Ismailov, F. I., Akhundov, G. A., and Vernich, O. R., 1966, Phys. Stat. sol., 17, K237.

Kuhn, A., Chevy, A., and Chevalier, R., 1975, Phys. Stat. sol. (a), 31, 469.

Leyris, J. P., Aicardi, J. P., and Soule, S., 1983, J. Lumin., 28, 65.

Manfredotti, C., Mancini, A. M., Murri, R., Rizzo, A., and Vasanelli, L., 1977, Nuovo Cim. B, 39, 257.

Manfredotti, C., Rizzo, A., De Blasi, C., Galassini, S., and Rugierro, L., 1975, J. appl. Phys., 46, 4531.

Mercier, A., and Voitchovsky, J. P., 1975, J. Phys. Chem. Solids, 36, 1411.

Montie, E. A., and van Gurp, G. J., 1989, J. appl. Phys., 66, 5549.

Pankove, J. I., 1971, Optical Processes in Semiconductors (Englewood Cliffs, New Jersey: Prentice-Hall), p. 150.

Poure, A., Leyris, J. P., and Aicardi, J. P., 1981, J. Phys. C, 14, 521.

Schmid, Ph., Voitchovsky, J. P., and Mercier, A., 1974, Phys. Stat. sol. (a), 21, 443.

Schmidt, T., Lischka, K., and Zulehner, W., 1992, Phys. Rev. B, 45, 8989.

Shigetomi, S., Ikari, T., and Nakashima, H., 1991, J. appl. Phys., 69, 7936; 1993, J. appl. Phys., 74, 4125; 1997, Phys. Stat. sol. (a), 160, 159.

Tagiev, B., Niftiev, G. M., and Abushov, S. A., 1984, Phys. Stat. Sol. (b), 121, K195.

Tagiev, B., Niftiev, G. M., and Aidaev, F. S., 1985, Phys. Stat. sol. (b), 128, K65.

Tatsuyama, C., Hamaguchi, C., Tomita, H., and Nakai, J., 1971, Jap. J. appl. Phys., 10, 1698.

Voitchovsky, J. P., and Mercier, A., 1974, Nuovo Cim. B, 22, 273.

Yu, P., and Cardona, M., 1995, Fundamentals of Semiconductors (Berlin: Springer), p. 348.

Zacks, E., and Halperin, A., 1972, Phys Rev. B, 6, 3072. 